\title{
Accuracy of automatic abnormal potential annotation for substrate identification in scar-related ventricular tachycardia
}

Yosuke Nakatani ${ }^{1}$, Philippe Maury $^{2}$, Anne Rollin $^{2}$, F. Daniel Ramirez ${ }^{3}$, Cyril Goujeau ${ }^{4}$, Takashi Nakashima ${ }^{5}$, Clémentine André ${ }^{6}$, Aline Carapezzi ${ }^{7}$, Philipp Krisai ${ }^{8}$, Takamitsu Takagi $^{9}$, Tsukasa Kamakura ${ }^{10}$, Konstantinos Vlachos ${ }^{11}$, Ghassen Cheniti ${ }^{12}$, Romain Tixier $^{13}$, Quentin Voglimacci-Stefanopoli ${ }^{14}$, Nicolas Welte ${ }^{15}$, Remi Chauvel ${ }^{16}$, Josselin Duchateau $^{13}$, Thomas Pambrun ${ }^{17}$, Nicolas Derval ${ }^{18}$, Mélèze Hocini1 ${ }^{19}$, Michel Haissaguerre $^{20}$, Pierre Jais ${ }^{13}$, and Frederic Sacher ${ }^{21}$

${ }^{1}$ University of Toyama

${ }^{2}$ University Hospital Rangueil

${ }^{3}$ Centre Hospitalier Universitaire de Bordeaux Hopital Cardiologique

${ }^{4}$, Service de Rhythmologie, Hôpital Cardiologique du Haut-Lévêque (Centre Hospitalier Universtaire de Bordeaux)

${ }^{5} 1$. Electrophysiology and Ablation Unit and L'Institut de rythmologie et modélisation cardiaque (LIRYC)

${ }^{6} \mathrm{CHU}$ Trousseau

${ }^{7}$ Boston scientific

${ }^{8}$ University of Bordeaux

${ }^{9}$ Yokosuka Kyosai Hospital

${ }^{10}$ National Cerebral and Cardiovascular Center

${ }^{11}$ Evangelismos General Hospital of Athens

${ }^{12}$ Hôpital Cardiologique du Haut Lévêque

${ }^{13}$ Centre Hospitalier Universitaire de Bordeaux

${ }^{14}$ University Hospital Rangueil, Toulouse

${ }^{15}$ Centre Hospitalier Universitaire de Bordeaux Hôpital Haut-Lévêque

${ }^{16}$ Hôpital Cardiologique du Haut-Lévêque (Centre Hospitalier Universtaire de Bordeaux)

${ }^{17}$ Hopital du Haut Leveque/LIRYC, Bordeaux

${ }^{18}$ Hopital cardiologique du haut-leveque

${ }^{19}$ Hôpital Cardiologique du Haut-Lévèque

${ }^{20}$ Hopital Cardiologique du Haut-Leveque

${ }^{21}$ Bordeaux University Hospital

February 27, 2021

\begin{abstract}
Introduction: Ultra-high-density mapping for ventricular tachycardia (VT) is increasingly used. However, manual annotation of local abnormal ventricular activities (LAVAs) is challenging in this setting. Therefore, we assessed the accuracy of the automatic annotation of LAVAs with the Lumipoint algorithm of the Rhythmia system (Boston Scientific). Methods and Results: One hundred consecutive patients undergoing catheter ablation of scar-related VT were studied. Areas with LAVAs
\end{abstract}


and ablation sites were manually annotated during the procedure and compared with automatically annotated areas using the Lumipoint features for detecting late potentials (LP), fragmented potentials (FP), and double potentials (DP). The accuracy of each automatic annotation feature was assessed by re-evaluating local potentials within automatically annotated areas. Automatically annotated areas matched with manually annotated areas in 64 cases (64\%), identified an area with LAVAs missed during manual annotation in 15 cases (15\%), and did not highlight areas identified with manual annotation in 18 cases (18\%). Automatic FP annotation accurately detected LAVAs regardless of the cardiac rhythm or scar location; automatic LP annotation accurately detected LAVAs in sinus rhythm, but was affected by the scar location during ventricular pacing; automatic DP annotation was not affected by the mapping rhythm, but its accuracy was suboptimal when the scar was located on the right ventricle or epicardium. Conclusion: The Lumipoint algorithm was as/more accurate than manual annotation in $79 \%$ of patients. FP annotation detected LAVAs most accurately regardless of mapping rhythm and scar location. The accuracy of LP and DP annotations varied depending on mapping rhythm or scar location.

\section{Hosted file}

VT lumipoint study Manuscript.pdf available at https://authorea.com/users/315801/articles/ 511082-accuracy-of-automatic-abnormal-potential-annotation-for-substrate-identificationin-scar-related-ventricular-tachycardia 